\title{
Therapie intraartikulärer Kalkaneusfrakturen
}

ie Behandlung der intraartikulären Fersenbeinfrakturen hat seit $2 \mathrm{Jahr}$ zehnten deutliche Verbesserungen im Management und in der Expertise gebracht.

Dennoch stehen verschiedene Fragen und Diskussionspunkte im Raum. Welche Frakturen sollen konservativ behandelt werden, welche offen operiert, und was für andere Optionen bestehen noch? Wie sieht ein Behandlungsalgorithmus unter Einbeziehung der Frakturtypen und Weichteilschäden sowie des Gesamtzustands des Patienten aus?

Aus Sicht der vorliegenden Studien gibt es hierauf keine schlüssigen Antworten. $Z u$ verschieden waren die angewendeten Methoden, die Evaluationsund Scoringsysteme, und zu verschieden waren die Definitionen, was eine anatomische Rekonstruktion, was ein gutes, befriedigendes oder ein schlechtes Ergebnis ist.

Die Traumatologie muß für sich in Anspruch nehmen, daß sie es über Jahre versäumt hat, das Problem stringent wissenschaftlich aufzuarbeiten.
Die Literatur weist eindeutig auf, daß erst von Thodarson 1996 eine prospektiv-randomisierte Studie durchgeführt wurde, die einem aussagekräftigen wissenschaftlichen Profil entspricht. Die Durchsicht unserer Langzeitergebnisse zeigt, daß die intraartikuläre Kalkaneusfraktur eine komplexe Gelenkfraktur ist, die nur bei anatomischer Rekonstruktion des gesamten Kalkaneus langfristig zu einem guten Ergebnis führt, und daß nur ein in der Kalkaneuschirurgie erfahrener Chirurg in der Lage ist, schwierige Frakturformen anatomisch zu rekonstruieren. Verschiedene Techniken zur Optimierung der Frakturreposition wurden zwischenzeitlich entwickelt (Atkens transkalkanearer Zugang, Zwipps subtalare Arthroskopie, Thermanns posteromediales, anterolaterales Fenster). Diese Entwicklungen zeugen davon, daß trotz des erweiterten lateralen Zugangs die Übersicht für eine anatomische Reposition nicht immer perfekt war.

Eine wissenschaftliche Aufarbeitung in den kommenden Jahren setzt jedoch eine Konsensusfindung für Klassifikations- und Evaluationssysteme voraus.

In der Literatur hat die SandersKlassifikation momentan den höchsten Stellenwert. Abzuwarten bleibt die Akzeptanz für das neue AO-Klassifika- tionssystem. Für das klinische Scoring hat sich im Fuß- und Sprunggelenkbereich immer mehr das Ankle/HindfootScoringsystem der „American Orthopedic Foot and Ankle Society" durchgesetzt.

Gelingt es mit einer einheitlichen "Sprache", prospektive Studien in einem Mindestzeitraum von 5 Jahren durchzuführen, werden wir vielleicht in Zukunft die Frage, wie die verschiedenen Frakturformen behandelt werden sollten, eindeutiger beantworten können.

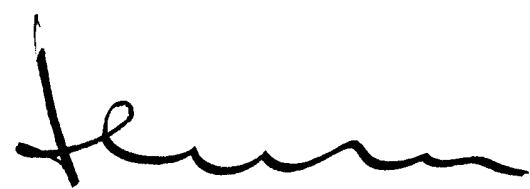

H. Thermann

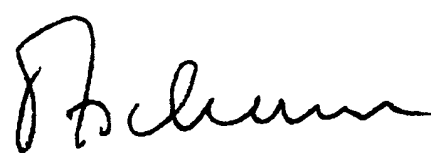

H. Tscherne 